STRUCTURAL

CHEMISTRY

ISSN 2053-2296

\title{
A smorgasbord of halogen bonds?
}

\author{
Gautam R. Desiraju*
}

Solid State and Structural Chemistry Unit, Indian Institute of Science, Bangalore 560 012, India. *Correspondence e-mail: gautam.desiraju@gmail.com

Keywords: halogen bond; iodine; crystal engineering.

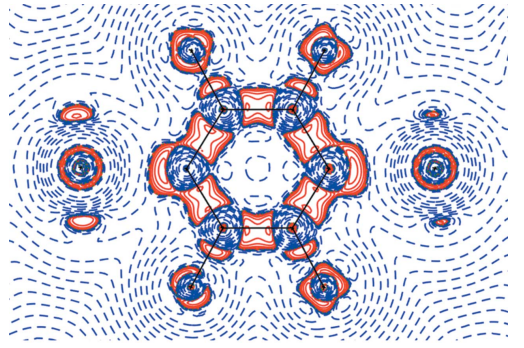

(C) 2019 International Union of Crystallography
In the beginning, hydrogen bonds were supposedly simple (Hüttermann, 2019), but life got more interesting as time went along. Who would have thought, for example, 100 years ago, when the hydrogen bond was first invoked, that we would be discussing $\mathrm{C}-\mathrm{H} \cdots \pi$ interactions (Nishio et al., 1998), in acetylene and benzene, and that we would be calling one of them a hydrogen bond and the other a van der Waals interaction? And what does one make of dihydrogen bonds? In the past, no one even knew that they existed (Bakhmutov, 2008).

In fact, this latter example is one of the rare cases where a halogen bond, of say the type $\mathrm{Cl} \cdots \mathrm{Cl}$, was identified and recognized long before its hydrogen-bond analog, i.e. $\mathrm{H} \cdots \mathrm{H}$, was even thought of. It just goes to show, and in part it is because of such thought exchanges, that there has been a tendency to liken halogen bonds to hydrogen bonds. This simplification certainly helped in the formal definition of the term 'halogen bond' where it was held that an electrophilic halogen atom is a sine qua non for a true halogen bond (Desiraju et al., 2013). But, on the other hand, this kind of comparison has also led to an inevitable tendency to think of the halogen bond as some sort of hydrogen bond with a halogen atom instead of the hydrogen. This is not quite accurate, and this is why we titled a review paper we wrote in 2013, 'Halogen bonds in crystal engineering: like hydrogen bonds yet different' (Mukherjee et al., 2014).

We tend to think of many intermolecular interactions in terms of electrostatics, but we need also to think of atom size. Halogen atoms, especially iodine, the one under discussion in an article by Wang et al. (2019) in the September issue of Acta Crystallographica Section $C$, can become quite large, and steric factors apart, this means that the degree of symmetry in the electronic distribution within an atom can be quite varied. The authors of the paper under consideration here have described, in fair detail, the chargedensity and spectroscopic properties of a pair of $\mathrm{I} \cdots \mathrm{N}$-containing cocrystals of $1,2,4,5$ tetrafluoro-3,6-diiodobenzene with two different $\mathrm{N}$-bases. The $\mathrm{I} \cdots \mathrm{N}$ distances are practically the same but the charge-density properties are different. Generally, the shorter a hydrogen bond gets, the stronger it gets. In this connection, one hears occasionally of breakdowns in this length-strength analogy in crystals (Braga et al., 1998), but in these cases there seem to be other extenuating circumstances. In the pair of cocrystals described here, however, there is a clear breakdown of this analogy for the $\mathrm{I} \cdots \mathrm{N}$ halogen bonds, at least if one goes by the charge-density results. The interactions are of nearly equal length, but as far as bond critical point (bcp) characteristics go, they would appear to be of differing strengths. It is shown that the use of another technique, in this case Raman spectroscopy, provides a clearer picture of this complex interaction. Perhaps the bcp alone is not a complete enough descriptor of halogen-bond strength.

The differences between the two title compounds have mainly to do with the polarization of the I-atom electron density in the two cases. Iodine is very different from the other halogens. The authors make an interesting comment, almost in passing, that bromine might not be a good via media between chlorine and iodine when one discusses halogen bonds, although this statement is made in the context of halogen-atom diffraction. In a general sense though, I have found this not to be so - bromine in fact did provide a good enough approximation, but possibly this issue will be better resolved in the future.

The key feature here is that the two halogen-bond acceptor sites, an aromatic nitrogen in 4-(dimethylamino)pyridine and an alicyclic nitrogen in DABCO, differ greatly. They differ appreciably in their basicity and hence will have distinct effects on the highly polarizable iodine even at nearly equal interatomic distances. Furthermore, their differing chemical situation will also render them to be of differing polarizability. The 
DABCO $\mathrm{N}$ atom possesses greater polarizing power than the pyridine $\mathrm{N}$ atom; iodine is highly polarizable additionally and therefore there is a skewed electronic distribution on the I atom, leading to a so-called $\sigma$-hole in the DABCO case. The Laplacian values in the two cases also point towards a higher covalent character in the pyridine case than in the DABCO case. This is reflected in the $V / G$ ratios mentioned in the article. Theoretical calculations generally fail to describe this effect properly due to a poor description of the electronic distribution for a heavy atom like iodine, but this is recognized by the authors. The high polarizability of iodine when compared to the other halogens is the critical attenuating factor which is responsible for the effects seen in the electron-density distributions in the two cases. Possibly such an effect might not be seen so easily for the corresponding $\mathrm{Br} \cdots \mathrm{N}$ interactions.

This article is worth highlighting because it opens up new opportunities in the charge-density and crystal engineering fields and in the overlap region between these fields. There is an urgent need to improve our charge-density modelling capabilities for heavy atoms. The lack of such a capability will hinder our progress in understanding halogen bonds. A great deal more needs to be done in terms of just reporting a greater variety of halogen bonds: our data bank of halogen bonds is still too small. The present paper is a one-off result and of course iodine is a special case. I would really like to see a lot more done with chlorine, which I personally believe to be the most challenging halogen with respect to the study of halogen bonds. The authors do not say much on the nature of the $(3,-1)$ bcp versus $\mathrm{Cl} \cdots \mathrm{Cl}$ distance in terms of the type-I and type-II classification (Desiraju \& Parthasarathy, 1989). Do the shorter contacts correspond mostly to type-II?

In the end, even hydrogen bonds are complex interactions: one needs to deal with electrostatics, covalency, polarization and dispersion before one begins to come to grips with the beast itself (Desiraju, 2002). The chances are that one is dealing with the same quartet of effects in a halogen bond, and it is likely that one needs many more examples before one is able to come up with a more complete description of the halogen bond. But in the end, we took about 100 years to get where we got with the hydrogen bond - and so we probably need to spend a little more time studying its halogen atom equivalent.

\section{Acknowledgements}

I thank T. S. Thakur (CSIR-CDRI, Lucknow) for helpful discussions. DST is acknowledged for support in the form of a J. C. Bose Fellowship

\section{References}

Bakhmutov, V. I. (2008). In Dihydrogen Bonds. Principles, Experiments and Application. New York: Wiley-Interscience.

Braga, D., Grepioni, F., Tagliavini, E., Novoa, J. J. \& Mota, F. (1998). New. J. Chem. 22, 755-757.

Desiraju, G. R. (2002). Acc. Chem. Res. 35, 565-573.

Desiraju, G. R., Ho, P. S., Kloo, L., Legon, A. C., Marquardt, R., Metrangolo, P., Politzer, P., Resnati, G. \& Rissanen, K. (2013). Pure Appl. Chem. 85, 1711-1713.

Desiraju, G. R. \& Parthasarathy, R. (1989). J. Am. Chem. Soc. 111, 8725-8726.

Hüttermann, A. (2019). In The Hydrogen Bond. A Bond for Life. Berlin: De Gruyter.

Mukherjee, A., Tothadi, S. \& Desiraju, G. R. (2014). Acc. Chem. Res. 47, 2514-2524.

Nishio, M., Hirota, M. \& Umezawa, Y. (1998). In The $C H / \pi$ Interaction. Evidence, Nature, and Consequences. New York: Wiley-VCH.

Wang, R., George, J., Potts, S. K., Kremer, M., Dronskowski, R. \& Englert, U. (2019). Acta Cryst. C75, 1190-1201. 